generic questionnaires (HAQ-DI and SF-36) and a disease-specific measurement RAQOL.

Methods: A total of 220 patients with a mean age $55.05 \pm 10.63$ SD meeting the 1987 ACR classification criteria for RA were included in the study. The mean disease duration was $9.97 \pm 5.78 \mathrm{SD}$. Patients were stratified according to treatment regimens into 2 age-matched treatment groups: 96 on csDMARDs and 124 on bDMARD therapy. Subjects with significant comorbidity, infectious disease, congestive heart failure (NYHA class III or IV), malignant hypertension, psychiatric illness, a history of lymphoproliferative disease or neoplasia were excluded from the study. All participants completed the HAQ-DI, SF-36v2TM and RAQoL at baseline, at months 6 and 12 thereafter. The scores of the three instruments were calculated via licensed calculator. Comparison was performed by analysis variance ANOVA.

Results: At baseline the mean scores of HAQ-DI and RAQoL did not differ greatly among patients on csDMARDs and bDMARDs $(1.29 \pm 0.78 S D$ vs $1.13 \pm 0.54 S D$, $p=0.063 ; 16.31 \pm 8.26 S D$ vs $15.03 \pm 7.13 S D, p=0.219$, respectively). However, the mean physical component summary score of SF-36 was significantly higher in bDMARDs compared with csDMARDs $(32.98 \pm 5.97$ vs $31.05 \pm 7.82$, $\mathrm{p}=0.039$ ), while in the mental component of this scoring system not such a difference was found $(p=0.983)$. After 6 months subjects treated with bDMARD showed a significant decreasing of the means of the HAQ-DI and RAQoL, as opposed to the other treatment group $(0.86 \pm 0.5 S D$ vs $1.17 \pm 0.76 S D, p<0.001$; $10.98 \pm 6.53 S D$ vs $14.55 \pm 7.96 S D, p<0.001$ respectively). Similar results were obtained for both physical and mental component summary scores of SF-36 (39.49 $\pm 6.43 S D$ vs $33.48 \pm 8.04 S D, p<0.001 ; 43.69 \pm 7,99 S D$ vs $39.66 \pm 10.19 S D$, $\mathrm{p}=0.001$ respectively). At month 12 a significant improvement of $\mathrm{QoL}$, measured by the three assessment tools was registered in patient receiving bDMARDs compared with the csDMARD treatment group $(p<0.001)$.

Conclusions: Patient treated with bDMARDs showed better results for QoL than those on therapy with csDMARDs within a period of 12 months of treatment. Current management strategies should focus on improving the symptoms of activity and maintaining physical function in order to increase QoL in patients with RA.

Disclosure of Interest: None declared

DOI: 10.1136/annrheumdis-2017-eular.3538

\section{AB0284 ASSESSMENT OF DISEASE ACTIVITY BY DAS28-CRP, CDAI, SDAI AND RESPONSE TO TREATMENT WITH CSDMARDS AND BDMARDS AFTER ONE-YEAR FOLLOW-UP IN RHEUMATOID ARTHRITIS PATIENTS FROM BULGARIAN POPULATION}

V.V. Boyadzhieva, N. Stoilov, M. Ivanova, R. Stoilov, R. Rashkov. Rheumatology, University Hospital "St. Iv. Rilski" Medical Faculty - Medical University, Sofia, Bulgaria

Background: The assessment of disease activity is an essential component in the selection of therapeutic approach for the prevention of disability of patients with RA.

Objectives: The current study was conducted to evaluate the disease activity in patients on csDMARDs and bDMARDs after 6 months to 1-year of treatment and to determine whether the benefits of different therapies were sustained over time. Methods: For the purpose of the study were selected 220 patients with a mean age 55.05 $\pm 10.63 S D$ years, meeting the 1987 ACR classification criteria for RA. Patients were stratified according to treatment regimens into 2 age-matched treatment groups: 96 on csDMARDs and 124 on bDMARD therapy. Patient's assessment of disease related pain, global health and physician assessment of global health was made by visual analogue scale (VAS) - 100mm. Disease activity was the primary outcome domain. Independent joint assessor evaluate 28 joints on baseline, 6th and 12th month of the follow-up period. C-reactive protein (CRP) was used to measure the inflammation process. DAS28-CRP, CDAI and SDAI were calculated according to the standard formulas. Comparison was performed by analysis variance ANOVA.

Results: On baseline, patients on bDMARDs had a significantly higher mean timeaveraged 28 -joint disease activity score ( $5.03 \pm 0.84 S D$ vs. $4.35 \pm 1.20 S D, p<0,001$ ), CDAI $(25.06 \pm 7.32 S D$ vs. $20.83 \pm 10.53 S D, p<0.001)$ and SDAI $(28.27 \pm 8.74$ SD vs. $23.19 \pm 11.89 \mathrm{SD}, \mathrm{p}<0.001)$ compared to those on csDMARDs. On the 6th month in both groups (bDMARDS and csDMARDs) we found significant decrease in mean DAS28 $(p<0.001, p<0.001)$, although no significant difference in disease activity between the groups was measured by this indicator $(3.75 \pm 2.49 \mathrm{SD}$ vs $3.90 \pm 1.10 \mathrm{SD}, \mathrm{p}=0.566$ ). Patients on bDMARDs had significantly lower disease activity compared to those on csDMARDs after 6th and 12th month of treatment assessed by CDAI $(13.43 \pm 4.98 \mathrm{SD}$ vs $16.81 \pm 9.94 \mathrm{SD}, \mathrm{p}=0.001 ; 8.65 \pm 4.53 \mathrm{SD}$ vs $15.86 \pm 10.02 \mathrm{SD}, \mathrm{p}<0.001)$, and SDAI $(14.63 \pm 5.42 \mathrm{SD}$ vs $18.38 \pm 10.49 \mathrm{SD}$, $\mathrm{p}<0.001 ; 9.39 \pm 4.92 \mathrm{SD}$ vs $16.79 \pm 10.5 \mathrm{SD}, \mathrm{p}<0.001)$. Unlike results reported by DAS28-CRP which showed no change between the 6th and 12th month in patients receiving csDMARDs $(3.90 \pm 1.10 \mathrm{SD}, 3.82 \pm 1.12 \mathrm{SD}, \mathrm{p}=0.156)$ we observed a statistically significant difference in all three time intervals $(0,6$ th, 12 th month) of the follow up period regarding to CDAI and SDAI.

Conclusions: After a year prospective follow-up, therapy with biologic DMARDs results in sustained suppression - minimal disease activity assessed by DAS28CRP, CDAI and SDAI, compared to patients receiving DMARDs who had moderate disease activity according to these tools. The therapy with bDMARDs was superior to csDMARDs therapy for suppressing disease activity (assessed by DAS28-CRP,
CDAI and SDAI) of rheumatoid arthritis (RA) on 6th and 12th month of the follow-up period.

Disclosure of Interest: None declared

DOI: 10.1136/annrheumdis-2017-eular.6650

\section{AB0285 CLINICAL SIGNIFICANCE OF GLUCOCORTICOID-INDUCED TUMOR NECROSIS FACTOR RECEPTOR RELATED PROTEIN LIGAND (GITRL) IN RHEUMATOID ARTHRITIS: RELATION TO DISEASE ACTIVITY AND TREATMENT OUTCOME}

W.A. Hassan ${ }^{1}$, A.I. Mansour ${ }^{2} .{ }^{1}$ Rheumatology and Rehabilitation; ${ }^{2}$ Clinical and chemical pathology, Benha university, Benha, Egypt

Background: Glucocorticoid-induced tumor necrosis factor receptor related protein (GITR) is a member of the tumor necrosis factor receptor superfamily that is activated by its specific ligand (GITRL). GITR is mainly expressed in immature and mature T cells especially regulatory (Treg) cells (CD4+ CD25+) and effector T cells (CD25-) [1]. GITRL is mainly expressed in endothelial cells, dendritic cells, macrophages and B cells but not in T cells. GITR-GITRL system is known to have important regulatory role on inflammatory response and immune reactivity.

Objectives: This study aimed to measure serum and synovial fluid (SF) levels of GITRL in patients with recent onset rheumatoid arthritis (RA) before and after initiation of therapy and to evaluate the relationship between GITRL and RA clinical and laboratory characteristics, disease activity and response to therapy.

Methods: We measured GITRL in the serum $(n=48)$ and SF samples $(n=21)$ from 48 recent onset RA patients and in the serum from 20 healthy control $(n=20)$ at baseline and 6 months after initiation therapy with non-biological disease modifying anti-rheumatic drugs (DMARDS). In the patients Disease activity was calculated by the 28 joint counts (DAS28) and musculoskeletal ultrasound examination (MSUS) was performed at baseline and after 6 months using a 12-joint score (bilateral elbow, wrist, 2nd metacarpophalangeal (MCP), 3rd MCP, knee, ankle) [2]; immunoglobulin-M rheumatoid factor (IgM-RF) titre, anti-cyclic citrullinated peptide (anti-CCP) antibodies titre and C-reactive protein (CRP) levels were measured and the health assessment questionnaire (HAQ) score were recorded.

Results: Serum and SF GITRL levels were highly significantly increased in RA (39.38 $\pm 16.78 \mathrm{ng} / \mathrm{mL}$ and $30.6 \pm 16.79 \mathrm{ng} / \mathrm{mL}$ respectively) compared to serum level in the healthy controls $(10.3 \pm 5.46 \mathrm{ng} / \mathrm{mL})(\mathrm{p}<0.001)$. In RA patients, baseline serum and SF levels of GITRL significantly correlated with DAS28 ( $r=0.52$ and 0.56 respectively, $p<0.05)$, anti-CCP titres $(r=0.46$ and 0.51 respectively, $p<0.05)$, grey scale (GS) $(r=0.5$ and 0.52 respectively, $p<0.05)$ and power Doppler (PD) $(r=0.65$ and 0.68 respectively, $p<0.001)$ synovitis scores. Also, serum and SF levels of GITRL at 6 months follow up significantly correlated with the DAS28 $(r=0.42$ and 0.48 respectively, $p<0.05)$, GS score $(r=0.46$ and 0.51 respectively, $p<0.05)$, PD signal $(r=0.43$ and 0.45 respectively, $p<0.05)$. Logistic regression analysis showed that baseline serum levels of GITRL were predictive of follow up DAS 28 and PD synovitis score ( $p=0.009$ and 0.03 respectively).

Conclusions: Rheumatoid arthritis patients have significantly increased serum and synovial levels of GITRL that remarkably correlated with the DAS28 and MSUS parameters of inflammations suggesting that it could be a useful marker to reflect RA disease activity. GITRL could be a useful biomarker to predict treatment outcome in RA patients.

References:

[1] Kim SH, Youn J. Rheumatoid Fibroblast-like Synoviocytes Downregulate Foxp3 Expression by Regulatory T Cells Via GITRL/GITR Interaction. Immune Netw. 2012;12(5):217-21.

[2] Naredo E, Rodríguez M, Campos C, Rodríguez-Heredia JM, Medina JA, Giner E, et al. Validity, reproducibility, and responsiveness of a twelve-joint simplified power Doppler ultrasonographic assessment of joint inflammation in rheumatoid arthritis. Arthritis Rheum 2008;59(4):515-22.

Disclosure of Interest: None declared

DOI: 10.1136/annrheumdis-2017-eular.5476

\section{AB0286 14 CASES STUDY OF MACROPHAGE ACTIVATION SYNDROME (MAS) IN SYSTEMIC ONSET JUVENILE IDIOPATHIC ARTHRITIS}

X. Chen ${ }^{1}, \mathrm{H}$. Zeng ${ }^{2} .{ }^{1}$ Department of Pediatric Allergy, Immunology and Rheumatolog; ${ }^{2}$ Department of Pediatric Allergy, Immunology and Rheumatology, Guangzhou Women and Children's Medical Center, Guangzhou, China

Background: Macrophage activation syndrome (MAS) is a severe, potentially life-threatening syndrome.

Objectives: we aim to review the precipitating events,clinical features, treatment, and outcome of macrophage activation syndrome (MAS).Part patients were analysed the Polymorphisms of Perforin A91V (NCBI:SNP rs35947132) using special primers by polymerase chain reaction (PCR).

Methods: Retrospective review of cases of MAS from a prospectively collected database of children with autoimmune diseases from 2003 to 2008.

Results: Fourteen patients (nine boys) were considered to have evidence of MAS.The primary diagnosis was systemic onset juvenile idiopathic arthristis, with age ranged from 5 months to 12 years. No medication was identified as trigger. Eleven had infections prior to MAS,specific infectious agents were identified in four. High grade fever, new onset hepatosplenomegaly, lymphadenopathy, dysfunction 